\title{
Conhecimento de ergonomia e desordens osteomusculares entre estudantes de Odontologia
}

\author{
Knowledge of ergonomics and musculoskeletal disorders \\ among dental students
}

\author{
Alberto José Oliveira Vieira* \\ Claudionor Antônio Barbosa Julião Filho* \\ Ramon Targino Firmino** \\ Ana Flávia Granville-Garcia** \\ Valdenice Aparecida de Menezes ${ }^{* * * * *}$
}

\section{Resumo}

Objetivo: Este trabalho tem o objetivo de avaliar o conhecimento de estudantes de Odontologia sobre os princípios ergonômicos aplicados à prática clínica e de investigar sintomas associados às doenças ocupacionais. Materiais e método: tratou-se de um estudo transversal censitário, no qual foram entrevistados 164 estudantes do $3^{\circ}$ ao $9^{\circ}$ período do curso de Odontologia de uma universidade do município de Caruaru-PE. Foram aplicados dois questionários: um, com perguntas relativas às características sociodemográficas, conhecimentos sobre ergonomia, doenças ocupacionais na Odontologia e medidas preventivas; e o outro, o Nordic musculoskeletal questionnaire, para mensuração de sintomas osteomusculares. Para análise estatística foi usado o teste de correlação de Pearson ou o Exato de Fisher $(p<0,05)$. Resultados: a maioria dos pesquisados, $87,2 \%$, soube expressar parcialmente o conceito de ergonomia, exemplificou como principais doenças ocupacionais os distúrbios osteomusculares, 54,3\%, bem como destacou realizar medidas preventivas "às vezes" $(45,7 \%)$, sendo os alongamentos a principal prática em 53\% dos casos. O local mais acometido por sintomas osteomusculares foi a parte superior das costas em $46,3 \%$ das ocorrências. Houve diferença estatística entre os períodos dos estudantes $\left(3^{\circ}, 5^{\circ}, 7^{\circ}\right.$ e $\left.9^{\circ}\right)$ para as variáveis: definição de ergonomia, frequência de uso de medidas preventivas, principais doenças ocupacionais e localização anatômica da dor $(p<0,05)$. Houve tendência de pior desempenho e maiores frequências de distúrbios osteomusculares nos acadêmicos dos períodos finais do curso. Conclusões: o conhecimento dos estudantes foi insatisfatório e as regiões mais acometidas por sintomas associados às doenças ocupacionais foram: a parte superior e a inferior das costas e do pescoço. Procedimentos normativos devem ser adotados com o intuito de prevenir as doenças ocupacionais no ambiente acadêmico.

Palavras-chave: Engenharia humana. Doenças ocupacionais. Estudantes de Odontologia.

\section{Introdução}

A ergonomia estuda a relação entre o homem e seus meios, seus métodos e seu ambiente de trabalho ${ }^{1}$. É a ciência que aplica teoria, princípios, dados e métodos para compreensão de produtos e sistemas de trabalho, a fim de aperfeiçoar a segurança e o bem-estar humano, bem como o desempenho dos sistemas. Pode-se afirmar que a ergonomia objetiva modificar o processo de trabalho para adequar a atividade do serviço às características, às habilidades e às limitações de pessoas com vistas à sua atuação eficiente, confortável e segura ${ }^{2,3}$.

*** Professora Doutora do Programa de Pós-Graduação em Odontologia da Universidade Estadual da Paraíba (UEPB), Campina Grande, Paraíba, PB, Brasil.

**** Professora Doutora adjunta do curso de Odontologia da Associação Caruaruense de Ensino Superior (Asces), Caruaru, Pernambuco, PE, Brasil. 
Os cirurgiões-dentistas (CDs) por terem a peculiaridade de trabalhar na cavidade bucal, uma área de acesso restrito, e com visibilidade limitada, exercem invariabilidades posturais, as quais podem gerar condições insalubres de trabalho, tornando-os vulneráveis a riscos ocupacionais ${ }^{4-6}$.

Estudos apontam prevalência entre $60 \%$ e $93 \%$ de CDs que relataram desconforto, dores ou sofrer de algum tipo de lesão musculoesquelética, sendo o pescoço, o ombro, os membros superiores e, principalmente, a região lombar as partes anatômicas mais afetadas. Esses problemas são decorrentes das más posturas adotadas no exercício da profissão, pois os profissionais permanecem sentados durante horas, realizando movimentos repetitivos, forçando as estruturas articulares e musculares ${ }^{7,8}$.

Visando à correção ou à melhoria da atividade prática na clínica odontológica, a Ergonomia aplicada à Odontologia tem como finalidade desenvolver um ambiente ergonomicamente planejado, com equipamentos racionalmente distribuídos, para diminuir o estresse físico e cognitivo, prevenir as doenças relacionadas ao exercício profissional, buscando um aumento de produtividade, melhoria na qualidade do serviço prestado e diminuição da fadiga na equipe de trabalho $0^{9-11}$.

Estudantes de odontologia também são susceptíveis a desenvolverem distúrbios musculoesqueléticos ${ }^{12}$. Estudos prévios, conduzidos com essa população, demonstram pouco conhecimento sobre o tema, bem como serem frequentes posturas inadequadas durante realização de atendimento odontológico ${ }^{13-15}$. Portanto, um monitoramento precoce e constante dos aspectos ergonômicos dos estudantes é relevante, uma vez que desordens osteomusculares podem aparecer cedo na carreira odontológica e, com o passar do tempo, são mais difíceis de serem eliminadas ${ }^{5,16-18}$.

Nessa perspectiva, o objetivo ora proposto foi avaliar o conhecimento de estudantes de Odontologia sobre os princípios ergonômicos aplicados à prática clínica, além de investigar sintomas associados às doenças ocupacionais.

\section{Materiais e método}

Este trabalho foi aprovado pelo Comitê de Ética e Pesquisa da Associação Cararuense de Ensino Superior (CAAE: 15954413.0.0000.5203), de acordo com as disposições da resolução 466/12 do Conselho Nacional de Saúde.

Tratou-se de um estudo transversal censitário, realizado na Associação Cararuense de Ensino Superior (Asces), localizada na cidade de Caruaru, cidade do agreste de Pernambuco, região Nordeste do Brasil. Foi realizado um censo, entre os meses de junho a agosto de 2013, com 164 estudantes que estavam regularmente matriculados entre o segundo e o quinto anos do curso de Odontologia dessa instituição de ensino. Foram excluídos do estudo os estudantes que não cursaram o eixo temático que contempla o conteúdo de orientação profissional.

Para a coleta dos dados foram utilizados dois instrumentos, sendo um questionário com perguntas abertas e de múltipla escolha ${ }^{19}$, que continha questões relativas às características sociodemográficas (idade e gênero), nível acadêmico, conhecimentos relacionados ao conceito de ergonomia, doenças ocupacionais na Odontologia, medidas preventivas no combate às doenças ocupacionais e aplicabilidades clínicas das diretrizes ergonômicas na Faculdade.

O segundo instrumento consistiu da versão brasileira validada de um questionário, contendo quatro questões de múltipla escolha, desenvolvido para padronização da mensuração de sintomas osteomusculares (Nordic musculoskeletal questionnaire $)^{20}$. Esse questionário apresenta uma figura humana, dividida em nove regiões anatômicas: cervical, ombros, torácica, cotovelos, punhos/mãos, lombar, quadril/coxas, joelhos, tornozelos/pés.

Os dados foram analisados por meio de estatística descritiva (determinação de frequências) e inferencial, a partir do teste Qui-Quadrado de Pearson ou o teste Exato de Fisher quando as condições para utilização do primeiro não foram verificadas $(\mathrm{p}<0,05)$. O programa estatístico utilizado foi o Statistical Package for the Social Sciences (SPSS) na versão 21 (IBM SPSS Statistics para Windows, Versão 21.0. Armonk, NY: IBM Corp.).

\section{Resulltados}

A Tabela 1 demonstra os resultados relativos às características da amostra no grupo total pesquisado, segundo o período de estudo. A maior parte dos estudantes tinha idade entre 21 e 30 anos $(62,8 \%)$ e era do sexo feminino $(67,3 \%)$.

\begin{tabular}{|c|c|c|}
\hline Variável & $\mathrm{n}$ & $\%$ \\
\hline \multicolumn{3}{|l|}{ Sexo } \\
\hline Masculino & 51 & 32,7 \\
\hline Feminino & 105 & 67,3 \\
\hline Total $\left.\right|^{(1)}$ & 156 & 100,0 \\
\hline \multicolumn{3}{|l|}{ Faixa etária } \\
\hline Até 20 & 46 & 28,0 \\
\hline 21 a 30 & 103 & 62,8 \\
\hline 31 ou mais & 15 & 9,1 \\
\hline Total & 164 & 100,0 \\
\hline \multicolumn{3}{|l|}{ Período em curso } \\
\hline Terceiro & 40 & 24,4 \\
\hline Quinto & 40 & 24,4 \\
\hline Sétimo & 38 & 23,2 \\
\hline Nono & 46 & 28,0 \\
\hline Total & 164 & 100 \\
\hline
\end{tabular}

Fonte: dos autores.

(1): Para oito alunos pesquisados não se dispõe dessa informação. 
A maioria dos pesquisados, $87,2 \%$ souberam parcialmente definir ergonomia, havendo diferença estatística entre os períodos ( $\mathrm{p}<0,001)$, observando-se menores percentuais de respostas corretas com o decorrer do curso. Ao serem questionados sobre quais as principais doenças ocupacionais, $54,3 \%$ destacaram os problemas osteomusculares segui- dos por $48,8 \%$ que lembraram as lesões por esforço repetitivo (LER).

Houve diferença estatística entre os períodos nos itens "LER" e "problemas auditivos/visuais", os quais foram mais frequentemente indicados por acadêmicos dos últimos períodos (Tabela 2 ).

Tabela 2 - Avaliação do conhecimento sobre definição de ergonomia e principais doenças ocupacionais, segundo o período

\begin{tabular}{|c|c|c|c|c|c|c|c|c|c|c|c|}
\hline \multirow{3}{*}{ Variáveis } & \multicolumn{8}{|c|}{ Período } & \multirow{2}{*}{\multicolumn{2}{|c|}{ Grupo total }} & \multirow{3}{*}{ Valor de $p$} \\
\hline & \multicolumn{2}{|c|}{$3^{\circ}$} & \multicolumn{2}{|c|}{$5^{\circ}$} & \multicolumn{2}{|c|}{$7^{\circ}$} & \multicolumn{2}{|c|}{$9^{\circ}$} & & & \\
\hline & $\mathrm{n}$ & $\%$ & $\mathrm{n}$ & $\%$ & $\mathrm{n}$ & $\%$ & $\mathrm{n}$ & $\%$ & $n$ & $\%$ & \\
\hline \multicolumn{12}{|l|}{ Definição de ergonomia } \\
\hline Correta & 4 & 10,0 & - & - & 2 & 5,3 & 2 & 4,3 & 8 & 4,9 & $\mathrm{p}^{(1)}<0,001^{*}$ \\
\hline Parcialmente correta & 27 & 67,5 & 37 & 92,5 & 35 & 92,1 & 44 & 95,7 & 143 & 87,2 & \\
\hline Incorreta & 9 & 22,5 & 3 & 7,5 & 1 & 2,6 & - & - & 13 & 7,9 & \\
\hline \multicolumn{12}{|l|}{ Doenças ocupacionais } \\
\hline Osteomusculares & 19 & 47,5 & 17 & 42,5 & 25 & 65,8 & 28 & 60,9 & 89 & 54,3 & $\mathrm{p}^{(2)}=0,121$ \\
\hline LER & 12 & 30,0 & 11 & 27,5 & 18 & 47,4 & 39 & 84,8 & 80 & 48,8 & $\mathrm{p}^{(2)}<0,001 *$ \\
\hline Problemas auditivos / Visuais & 7 & 17,5 & 1 & 2,5 & 3 & 7,9 & 11 & 23,9 & 22 & 13,4 & $\mathrm{p}^{(2)}=0,018^{*}$ \\
\hline
\end{tabular}

\section{Fonte: dos autores.}

$\left.{ }^{*}\right)$ : Diferença significativa ao nível de $5 \%$.

(1): Por meio do teste Exato de Fisher.

(2): Por meio do teste Qui-Quadrado de Pearson.

No tocante à frequência de utilização de medidas preventivas para o combate a doenças ocupacionais, quase metade dos sujeitos respondeu que 0 fazia às vezes, $45 \%$. Observa-se diferença significativa entre os períodos $(p=0,010)$, havendo uma ten- dência ao aumento da frequência com o avançar do curso. Em relação às medidas preventivas, a realização de alongamentos foi a atitude mais realizada em 53\%, não havendo diferença estatística entre os períodos (Tabela 3).

Tabela 3 - Avaliação do uso e da frequência de uso de medidas preventivas no combate às doenças ocupacionais, segundo o período

\begin{tabular}{|c|c|c|c|c|c|c|c|c|c|c|c|}
\hline \multirow{3}{*}{$\begin{array}{l}\text { Uso de medidas preventivas } \\
\text { para doenças ocupacionais }\end{array}$} & \multicolumn{8}{|c|}{ Período } & \multirow{2}{*}{\multicolumn{2}{|c|}{ Grupo total }} & \multirow{3}{*}{ Valor de $p$} \\
\hline & \multicolumn{2}{|c|}{$3^{\circ}$} & \multicolumn{2}{|c|}{$5^{\circ}$} & \multicolumn{2}{|c|}{$7^{\circ}$} & \multicolumn{2}{|c|}{$9^{\circ}$} & & & \\
\hline & $\mathrm{n}$ & $\%$ & $\mathrm{n}$ & $\%$ & $\mathrm{n}$ & $\%$ & $\mathrm{n}$ & $\%$ & $\mathrm{n}$ & $\%$ & \\
\hline Nenhuma & 13 & 32,5 & 9 & 22,5 & 14 & 36,8 & 9 & 19,6 & 45 & 27,4 & $\mathrm{p}^{(1)}=0,010^{*}$ \\
\hline Às vezes & 22 & 55,0 & 24 & 60,0 & 13 & 34,2 & 16 & 34,8 & 75 & 45,7 & \\
\hline Raramente & 1 & 2,5 & 3 & 7,5 & 8 & 21,1 & 13 & 28,3 & 25 & 15,2 & \\
\hline Diariamente & 3 & 4,0 & 8 & 4,0 & 21 & 3,0 & 28 & 8,0 & 15 & 19,0 & \\
\hline Medidas preventivas & & & & & & & & & & & \\
\hline Exercícios regulares & 19 & 47,5 & 21 & 52,5 & 11 & 28,9 & 16 & 34,8 & 67 & 40,9 & $\mathrm{p}^{(1)}=0,116$ \\
\hline Alongamentos & 23 & 57,5 & 17 & 42,5 & 18 & 47,4 & 29 & 63,0 & 87 & 53,0 & $\mathrm{p}^{(1)}=0,217$ \\
\hline RPG/ Pilates & 9 & 22,5 & 9 & 22,5 & 10 & 26,3 & 15 & 32,6 & 43 & 26,2 & $p^{(1)}=0,672$ \\
\hline Outras & 1 & 2,5 & - & - & 2 & 5,3 & 1 & 2,2 & 4 & 2,4 & $p^{(1)}=0,546$ \\
\hline
\end{tabular}

Fonte: dos autores.

${ }^{*}$ ): Diferença significativa ao nível de $5 \%$.

(1): Por meio do teste Qui-Quadrado de Pearson.

As regiões anatômicas com mais relatos de sintomas osteomusculares nos últimos 12 meses foram, respectivamente, a parte superior das costas, com $46,3 \%$, a inferior das costas $40,2 \%$ e o pescoço
$40,2 \%$. Houve diferença significativa para o item "pescoço" $(p=0,02)$, no qual os relatos de incômodo aumentaram com o avançar do curso, como se pode ler na Tabela 4. 
Tabela 4 - Região anatômica em relação à prevalência de sintomas osteomusculares nos últimos 12 meses, segundo o período

\begin{tabular}{|c|c|c|c|c|c|c|c|c|c|c|c|}
\hline \multirow{3}{*}{ Região } & \multicolumn{8}{|c|}{ Período } & \multirow{2}{*}{\multicolumn{2}{|c|}{ Grupo total }} & \multirow{3}{*}{ Valor de $\mathrm{p}$} \\
\hline & \multicolumn{2}{|c|}{$3^{\circ}$} & \multicolumn{2}{|c|}{$5^{\circ}$} & \multicolumn{2}{|c|}{$7^{\circ}$} & \multicolumn{2}{|c|}{$9^{\circ}$} & & & \\
\hline & $\mathrm{n}$ & $\%$ & $\mathrm{n}$ & $\%$ & $\mathrm{n}$ & $\%$ & $\mathrm{~N}$ & $\%$ & $\mathrm{~N}$ & $\%$ & \\
\hline Pescoço & 8 & 20,0 & 15 & 37,5 & 15 & 39,5 & 28 & 60,9 & 66 & 40,2 & $\mathrm{p}^{(1)}=0,002^{*}$ \\
\hline Ombros & 11 & 27,5 & 13 & 32,5 & 12 & 31,6 & 21 & 45,7 & 57 & 34,8 & $p^{(1)}=0,309$ \\
\hline Parte superior das costas & 16 & 40,0 & 16 & 40,0 & 18 & 47,4 & 26 & 56,5 & 76 & 46,3 & $p^{(1)}=0,358$ \\
\hline Cotovelos & 3 & 7,5 & 1 & 2,5 & - & - & 4 & 8,7 & 8 & 4,9 & $p^{(2)}=0,222$ \\
\hline Punhos/mãos & 9 & 22,5 & 16 & 40,0 & 17 & 44,7 & 17 & 37,0 & 59 & 36,0 & $p^{(1)}=0,193$ \\
\hline Parte inferior das costas & 14 & 35,0 & 12 & 30,0 & 17 & 44,7 & 23 & 50,0 & 66 & 40,2 & $p^{(1)}=0,227$ \\
\hline
\end{tabular}

Fonte: dos autores.

${ }^{\star}{ }^{\star}$ : Diferença significativa ao nível de $5,0 \%$

(1): Por meio do teste Qui-Quadrado de Pearson.

(2): Por meio do teste Exato de Fisher.

\section{Discussão}

Este estudo teve por objetivo determinar o nível de conhecimentos de acadêmicos de Odontologia sobre ergonomia, bem como investigar a presença de desordens osteomusculares em virtude da atividade odontológica nesses indivíduos. Os alunos geralmente não têm as habilidades físicas e a preparação psicológica necessária para exercer a profissão quando comparados a um dentista qualificado. Portanto, para reduzir o risco de desenvolvimento de lesões músculo-esqueléticas, é extremamente importante que o treinamento seja iniciado o mais cedo possível, enquanto esses ainda estão sendo academicamente "modelados" para a profissão, o que possibilitará que os alunos sejam capazes de identificar possíveis defeitos posturais e corrigí-los o quanto antes ${ }^{18,21}$.

De uma forma geral, os indivíduos ora pesquisados apresentaram conhecimentos insatisfatórios a respeito do tema estudado, sendo comum relatos de sintomas osteomusculares. Esses dados refletem que ainda existe uma carência na formação desses sujeitos, além de demonstrar que dentistas são propensos, desde o início de suas práticas clínicas como estudantes, a lesões do sistema músculo-esquelético em decorrência do exercício clínico da profissão ${ }^{22}$.

Apesar da existência de regulamentos específicos que normatizam as rotinas da prática odontológica, visando à otimização do trabalho e consequentemente a mais saúde, conforto e segurança para o trabalhador, ainda é alta a ocorrência de distúrbios osteomusculares entre os profissionais. Não há unanimidade na adoção de medidas preventivas no exercício clínico diário, tanto entre os estudantes de Odontologia quanto entre os cirurgiões-dentistas ${ }^{7-9,10,19,23}$.

Ergonomia é a disciplina científica relacionada com a compreensão das interações entre seres humanos e outros elementos de um sistema, a fim de otimizar o bem-estar humano e do sistema global de desempenho. Tópicos relevantes incluem posturas de trabalho, manuseio de materiais, movimentos repetitivos, distúrbios músculo-esqueléticos relacionados com o trabalho, com o layout do local de trabalho, com a segurança e com a saúde ${ }^{1}$. Nessa perspectiva, os participantes foram solicitados a definir ergonomia, sendo que $87,2 \%$ definiram de forma parcialmente correta, de modo semelhante aos achados de estudo prévio ${ }^{19}$. Neste caso, foram consideradas corretas as respostas que incluíram aspectos dos equipamentos e das posições no trabalho, bem como medidas e atitudes preventivas no combate às desordens ocupacionais. E, parcialmente correta, as respostas que citaram apenas posição ou postura adequada no ambiente de trabalho. Houve diferença significativa entre os períodos cursados pelos alunos $(p<0,001)$, no qual o percentual de respostas corretas diminuiu com o avançar do curso. Provavelmente, isso ocorreu pelo fato de os alunos terem contato com esses assuntos, principalmente de forma teórica, nos períodos iniciais da graduação, de maneira que o conhecimento não perdurou ao longo do curso, talvez por falta de exigência. Novas estratégias de ensino, que permitam uma retenção em longo prazo dos conhecimentos transmitidos, fazem-se necessárias.

Questionou-se aos participantes se eles conheciam alguma doença ocupacional. Cerca de 30\% afirmou não conhecer nenhum problema, enquanto que os demais pesquisados apontaram como principais agravos os distúrbios osteomusculares e as lesões por esforço repetitivo. Lesões por esforços repetitivos (LER) e/ou distúrbios osteomusculares relacionados ao trabalho (DORT) são síndromes que acometem um grande número de indivíduos e apresentam etiologia multifatorial, sendo, portanto, de grande complexidade diagnóstica ${ }^{24}$. Por isso, a importância do aprofundamento de seu estudo nas diversas idades cronológicas e exposições ambientais, o que favorece melhor suporte na formação dos profissionais da saúde e, consequentemente, na sua atuação $0^{25}$. Houve diferença estatística para os 
itens LER $(p<0,001)$ e problemas auditivos/visuais ( $p=0,028)$, os quais foram frequentemente mais indicados por acadêmicos dos últimos períodos. Isso pode ser explicado, provavelmente devido a uma maior vivência na prática clínica.

O exercício leva ao fortalecimento do sistema músculo-esquelético. O sistema reforçado resiste e tolera melhor maiores tempos em posições estáticas, comuns da prática odontológica ${ }^{12}$. Estudos, dentre esses, uma revisão sistemática, provaram que exercícios físicos regulares foram associados a uma diminuição da intensidade da dor e da fadiga durante a prática odontológica, sendo, tal prática correlacionada positivamente com redução na prevalência de lesões músculo-esqueléticas ${ }^{12,23}$. Na presente investigação, cerca de $30 \%$ dos pesquisados apontaram não utilizar nenhuma medida preventiva para os problemas ocupacionais, enquanto que $45 \%$ deles afirmaram realizar esporadicamente, sendo os alongamentos, as condutas realizadas com maior frequência (45\%), o que faz crer que seja essa uma maneira fácil e viável para a prevenção de desordens ocupacionais no cotidiano dos estudantes e dos profissionais. Houve diferença estatística no tocante à frequência de realização $(p=0,01)$, que tendeu a ser maior com o avançar do curso, provavelmente devido a um maior número de episódios de incômodo, em decorrência do aumento da carga horária vivenciada em ambiente clínico. Esses resultados assemelham-se a um estudo realizado com acadêmicos da Malásia, o qual evidenciou que $51 \%$ dos estudantes estavam familiarizados com os métodos preventivos, entretanto, apenas $20 \%$ realizavam alongamentos ${ }^{8}$. Resultados inferiores foram observados em investigação realizada com estudantes da Colômbia, na qual apenas $13 \%$ dos entrevistados relataram praticar alongamentos ${ }^{22}$. Esse fato chama a atenção para uma possível deficiência das instituições de ensino na orientação de medidas de prevenção antes e nos intervalos dos atendimentos clínicos, principalmente na fase de formação do estudante.

Quando se fala em doenças ocupacionais, vale salientar a qualidade de vida no ambiente de trabalho. A concretização e a aplicação dos princípios ergonômicos que vislumbrem a identificação e a modificação das inadequações posturais fazem-se necessárias, sendo uma maneira eficaz de se garantir a salubridade, a segurança, o alto desempenho, a motivação e a satisfação na prática odontológica. Em adição, novos modelos de saúde e de segurança do trabalho surgem para exigir dos profissionais um novo reposicionamento, com a introdução e o aprimoramento dos conhecimentos e das aplicabilidades ergonômicas, sobretudo nas universidades, enquanto instituições responsáveis pela formação dos futuros profissionais ${ }^{19,26,27}$.

A falta de um planejamento adequado do ambiente e do equipamento de trabalho favorece o surgimento de distúrbios no sistema osteomuscular principalmente nas regiões do pescoço, dos ombros, da parte superior e inferior das $\operatorname{costas}^{23}$. Essas regiões do corpo são mais susceptíveis a lesões, uma vez que a maioria dos estudantes, para uma melhor visão e acesso, eleva a cabeça, flexiona o pescoço e gira o ombro e o peito para uma posição instável e sem suporte assimétrico ${ }^{8,12}$.

Alterações nessas regiões anatômicas também foram frequentes neste estudo. Verificou-se, nos últimos 12 meses, que a parte superior e a parte inferior das costas, bem como o pescoço foram às regiões com percentuais mais elevados de sintomatologia dolorosa. Para esse último, observou-se diferença estatística entre os períodos $(p=0,002)$, com frequências crescentes com o avançar do curso. Outros estudos também encontraram resultados semelhantes ${ }^{8,12,22,23}$. É válido ressaltar que, de uma forma geral, a frequência de incômodos aumentou em todas as regiões anatômicas à medida que os períodos avançaram, comprometendo cerca de metade dos entrevistados no último semestre. Essa ocorrência possivelmente pode estar relacionada com 0 fato de que com o passar dos períodos os estudantes ficam mais sujeitos a distúrbios dessa natureza, pois exercem, de forma mais intensiva, suas atividades clínicas. Além disso, estudo prévio realizado com acadêmicos de Odontologia dessa mesma instituição de ensino demonstrou que todos os sujeitos pesquisados apresentaram posturas incorretas durante o atendimento clínico, o que pode ajudar a explicar nossos achados ${ }^{28}$. Adotar posturas adequadas na prática clínica e ter um ambiente de trabalho favorável pode reduzir a frequência de lesões para o sistema esquelético muscular, evitando uma aposentadoria precoce da profissão. Portanto, é de vital importância promover a formação de saúde ocupacional e programas de prevenção em relação a posturas ergonômicas, que devem ser adquiridas pelos estudantes de Odontologia durante suas práticas clínicas, favorecendo desse modo, estilos de vida saudáveis ${ }^{22}$. A alta prevalência de incômodo nas costas, no pescoço e nos ombros indicam claramente uma preocupação com o futuro da saúde no trabalho desse grupo ${ }^{23}$.

O presente estudo utilizou um questionário autoaplicável, o que pode ter introduzido um viés de resposta e limitar a generalização dos resultados. No entanto, esse aspecto foi minimizado a partir da utilização de um instrumento validado. Considerando o alto risco de desenvolvimento de lesões osteomusculares entre estudantes de Odontologia $^{12}$, orientações sobre os princípios ergonômicos e adoção de medidas preventivas devem ser implementadas desde o início do curso, pois ajudam a aumentar a consciência dos alunos sobre o tema, promovendo a sua própria saúde e mantendo a sua qualidade de vida ${ }^{18}$. 


\section{Conclusão}

O conhecimento dos estudantes pesquisados foi considerado insatisfatório e as regiões corporais mais acometidas por sintomas associados às doenças ocupacionais foram: parte superior e inferior das costas e do pescoço. É de suma importância que procedimentos normativos sejam adotados com o intuito de se prevenir as doenças ocupacionais no ambiente acadêmico, pois é preocupante o fato de estudantes, em pleno processo de formação, já apresentarem desordens dessa natureza e desconhecerem os princípios básicos de ergonomia.

\section{Abstract}

Objective: to evaluate the knowledge of Dentistry students on ergonomic principles applied to clinical practice, and to investigate symptoms associated with occupational diseases. Materials and Method: it was a cross-sectional census study, in which 164 students from the $3^{\text {rd }}$ to the $9^{\text {th }}$ school period of the Dentistry course at a University in the city of Caruaru, PE, Brazil, were interviewed. Two questionnaires were applied: one with questions pertaining to sociodemographic characteristics, knowledge about ergonomics, occupational diseases in Dentistry, and preventive measures; and the Nordic Musculoskeletal Questionnaire for measurement of musculoskeletal symptoms. Statistical analysis was performed using the Pearson's correlation test or Fisher's Exact test $(p<0.05)$. Results: most of the respondents (87.2\%) partially knew the concept of ergonomics, cited musculoskeletal disorders as the main occupational disorders (54.3\%), and emphasized performing preventive measures "sometimes" (45.7\%), considering stretching the most cited practice (53.0\%). The most frequently affected site of musculoskeletal symptoms was the upper back (46.3\%). There was statistical difference between the school periods of the students $\left(3^{\text {rd }}, 5^{\text {th }}, 7^{\text {th }}\right.$, and $\left.9^{\text {th }}\right)$ for the variables: definition of ergonomics, frequency of use of preventive measures, main occupational diseases, and anatomical site of pain $(p<0.05)$. There was a trend for worse performance and higher frequency of musculoskeletal disorders in students from the final periods of the academic course. Conclusions: the knowledge of students was unsatisfactory and regions most affected by symptoms associated with occupational diseases were upper and lower back and neck. Normative procedures must be followed in order to prevent occupational diseases in the academic environment.

Keywords: Human Engineering. Occupational Diseases. Dentistry Students.

\section{Referências}

1. International Ergonomics Association. Nordic Ergonomics Society. The Discipline of Ergonomics 2000. [Citado 2013 Mar 10]. Disponível em URL: http://www.nordiskergonomi.org.

2. Gopinadh A, Devi KNN, Chiramana S, Manne P, Sampath A, Babu MS. Ergonomics and musculoskeletal disorder: as an occupational hazard in dentistry. J Contemp Dent Pract 2013; 14(2):299-303.

3. Lombardi LR, Pizzol VD, Vidaurre G, Corletti RB, Barbosa RLF. Análise ergonômica do trabalho em uma serraria do estado do Espírito Santo. Floresta e Ambiente 2011; 18(3): 243-7.

4. Lindfors P, Von Thiele U, Lundberg U. Work Characteristics and upper extremity disorders in female Dental Health Workers. J Occup Health 2006; 48(3):192-7.

5. Garcia PPMS, Campos JADB, Zuanon ACC. Avaliação clínica das posturas de trabalho empregadas na clínica de odontopediatria por estudantes de Odontologia. Pesq Bras Odontoped Clin Integr 2008; 8(1):31-7.

6. Gupta S. Ergonomic applications to dental practice. Indian J Dent Res 2011; 22(6):816-22.

7. Harutunain K, Albiol JG, Figueiredo R, Escoda CG. Ergonomics and musculoskeletal pain among postgraduate students and faculty members of the School of Dentistry of the University of Barcelona (Spain). A cross-sectional study. Med Oral Patol Oral Cir Bucal 2011; 16(3):425-9.

8. Khan SA, Chew KY. Effect of working characteristics and taught ergonomics on the prevalence of musculoskeletal disorders amongst dental students. BMC Musculoskeletal Disorders 2013; 14: 118.

9. Kumar SP, Kumar V, Baliga M. Work-related musculoskeletal disorders among dental professionals: an evidencebased update. Indian Journal of Dental Education. 2012; 5(1): 5-12.

10. Presoto CD, Corrocher PA, Campos JADB, Garcia PPNS Fatores de risco de desordens osteomusculares presentes no posto de trabalho de estudantes de Odontologia. Pesq Bras Odontoped Clin Integr. 2012; 12(4): 549-54.

11. Garbin AJÍ, Garbin CAS, Diniz DG. Normas e diretrizes ergonômicas em Odontologia: O caminho para a adoção de uma postura de trabalho saudável. Rev odontol Univ Cid Sao Paulo 2009; 21(2): 155-61.

12. Movahhed T, Ajami B, Soltani M, Shakeri MT, Dehghani M Musculoskeletal pain reports among Mashhad dental students, Iran. Pak J Biol Sci 2013; 16(2):80-5.

13. Bendezú-Aguierre NV, Tapia EV, Mendonza LAA, Fonseca GV. Correlación entre nivel de conocimientos sobre posturas odontológicas ergonómicas, posturas de trabajo y dolor postural según zonas de respuesta, durante las prácticas clínicas de estudiantes en una Facultad de Estomatología. Rev estomatol hered 2006; 16(1): 26-32.

14. Garbin AJ, Garbin CA, Diniz DG, Yarid SD. Dental students' knowledge of ergonomic postural requirements and their application during clinical care. Eur J Dent Educ 2011; 15(1):31-5.

15. Pietrobon L, Filho GIR. Doenças de caráter ocupacional em cirurgiões- dentistas - um estudo de caso sobre cifoescoliose. RFO Passo Fundo 2010; 15(2):111-8.

16. Rising DW, Bennett BC, Hursh K, Plesh O. Reports of body pain in a dental student population. J Am Dent Assoc 2005; 136(1):81-6.

17. Garcia PPNS, Pinelli C, Derceli JDR, Campos JADB. Musculoskeletal disorders in upper limbs indental students: exposure level to risk factors. Braz J Oral Sci 2012; 11(2): 148-53. 
18. Corrocher PA, Presoto CD, Campos JA, Garcia PP. The association between restorative pre-clinical activities and musculoskeletal disorders. Eur J Dent Educ 2013; [Epub ahead of print].

19. Cunha CAC. Conhecimento sobre ergonomia no âmbito acadêmico. [Dissertação de Mestrado]. Natal: Universidade Federal do Rio Grande do Norte; 2011.

20. Barros ENC, Alexandre, NMC. Cross-cultural adaptation of the nordic musculoskeletal questionnaire. Int Nurs Rev 2003; 50(2): 101-8.

21. Biswas R, Sachdev V, Jindal V, Ralhan S. Musculoskeletal Disorders and Ergonomic Risk Factors in Dental Practice. Indian Journal of Dental Sciences 2012; 4(1): 70-4.

22. Diaz-Caballero AJ, Gómez-Palencia IP, Díaz-Cárdenas S. Ergonomic factors that cause the presence of pain muscle in students of dentistry. Med Oral Patol Oral Cir Bucal 2010; 15(6):906-11.

23. Hayes M, Cockrell D, Smith DR. A systematic review of musculoskeletal disorders among dental professionals. Int J Dent Hyg 2009; 7(3):159-65.

24. Alencar JF, Coury HJCG, Oishi J. Aspectos relevantes no diagnóstico de Dort e fibromialgia. Rev Bras Fisioter 2009; 13(1): 52-8.

25. Paixão MDS, Tassitano RM, Siqueira GRD. Prevalência de Desconforto Osteomuscular e Fatores Associados Em Estudantes Universitários. Rev Bras Promoç Saúde 2013; 26(2):242-50.

26. Dul J, Neumann WP. Ergonomics contributions to company strategies. Appl Ergon 2009; 40(4): 745-52.

27. Abarqhouei NS, Nasab HH. Total Ergonomics and Its Impact in Musculoskeletal Disorders and Quality of Work Life and Productivity. Open Journal of Safety Science and Technology. 2011; 1: 79-88.

28. Siqueira GR, Silva AM, Vieira RAG, Silva RB. Dores músculo-esqueléticas em estudantes de Odontologia. RBPS. 2010; 23(2): $150-9$

\section{Endereço para correspondência:}

Ana Flávia Granville-Garcia

Cap. João Alves Lira, no 1325/410 - Bela Vista

58101-281 Campina Grande - PB

E-mail: anaflaviagg@hotmail.com

Recebido: 25/05/2014. Aceito: 05/12/2014. 